Inhaltsverzeichnis/Contents

O. Knustad,

H. J. McQueen,

N. Ryum,

J. Solberg

M. Turwitt,

G. Elssner,

G. Petzow
A. Tekin,

A. Wirth

H. Asakura,

M. Takahara

F. K. Naumann,

F. Spies
Polarized Light Observation of Grain Extension and Subgrain Formation in Aluminium Deformed at $400{ }^{\circ} \mathrm{C}$ to Very High Strains

Polarisationsoptische Untersuchung der Kornstrekkung und der Subkornbildung von Aluminium nach hoher Verformung bei $400{ }^{\circ} \mathrm{C}$ 215

Metallographische Präparation von Metall/KeramikVerbindungen

Metallographic Preparation of Metal/Ceramic Composites

Persönliches/Personal Notes

The Structure and Mechanical Properties of Fe-Mn$\mathrm{Co}$ and Fe-Mn-Co-Mo Alloys (Part 1)

Gefüge und mechanische Eigenschaften von Fe-MnCo- und Fe-Mn-Co-Mo-Legierungen (Teil 1) 242

Preparation of Refractories by Automatic Polishing Automatische Präparation von feuerfesten Werkstoffen

Knüppel und Stangen aus Kugellagerstahl mit Innenfehlern

Billets and Bars of Ball Bearing Steel with Internal Defects

Tagungskalender/Meeting Diary 266 


\section{Metallographie -}

\section{Präparationsmethodik und Geftigeanalyse} metallischer und nichtmetallischer Werkstoffe

\section{Karin Dieser}

Wolf-Ulrich Kopp

Hermann Vetters
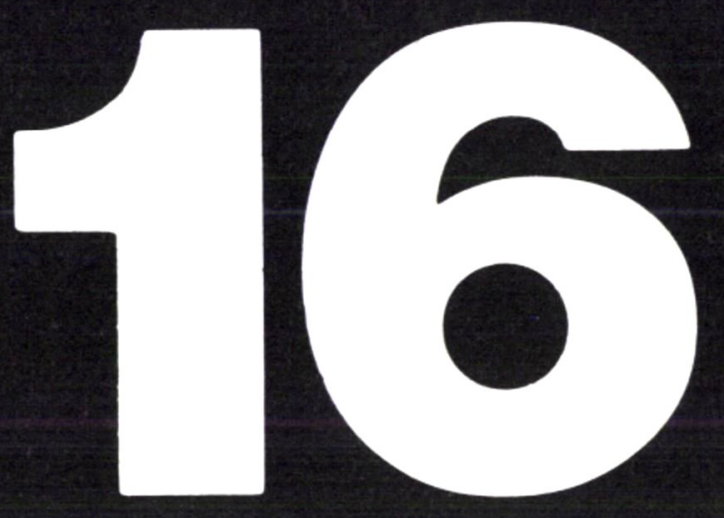

Berichte der Metallographie-Tagung Neu-UIm

26. bis 28. September 1984

\section{Veranstalter:}

Fachausschuß Metallographie

der Deutschen Gesellschaft für Metallkunde

Unterausschuß Metallographie im Werkstoffausschuß

des Vereins Deutscher Eisenhüttenleute

Institut für Metallkunde und Werkstoffprüfung

an der Montan-Universität Leoben

Technisch-Wissenschaftlicher Verein

Eisenhütte Österreich

Deutsche Keramische Gesellschaft

Dr. Riederer-Verlag GmbH, Stuttgart
NEU

Der Sympo-

sienband der MetallographieTagung, Berichte der MetallographieTagung Neu-Ulm, 26.-28. September 1984 ist jetzt erhältlich.

Sichern Sie sich Ihr Exemplar auf dem anhängenden Coupon zum Preis von DM 80,-

ca. 500 Seiten Hardcover

Format

$14,8 \times 21 \mathrm{~cm}$ Mit vielen Abbildungen und 9 Farbseiten

Die gesammelten Vorträge der MetallographieTagung in einem Band:

Sonderband 16

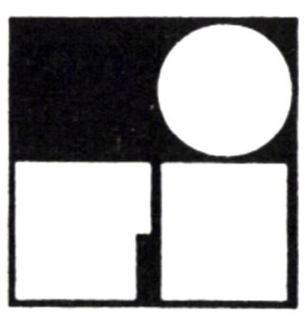

Dr. Riederer Verlag GmbH

Postfach 447 7000 Stuttgart 1 